\section{F214. PSYCHOLOGICAL INTERVENTIONS FOR POSITIVE SYMPTOMS IN SCHIZOPHRENIA: A NETWORK META-ANALYSIS}

\author{
Irene Bighelli*,1, Stefan Leucht ${ }^{1}$ \\ ${ }^{1}$ Technische Universität München
}

Background: There is rising awareness about the need of multi-disciplinary approaches integrating psychological treatments for schizophrenia, but a comprehensive evidence base on their relative efficacy is lacking. Conventional pairwise meta-analyses cannot provide a hierarchy based on the randomised evidence. We aimed to integrate the available evidence to create hierarchies of the comparative efficacy, acceptability and tolerability of psychological interventions for schizophrenia.

Methods: We performed a network meta-analysis (which uses both direct and indirect comparisons) of randomized controlled trials on psychological treatments aimed at positive symptoms in the acute treatment of schizophrenia, compared with another psychological intervention or with a no treatment condition (waiting list, treatment as usual). We excluded trials done in patients with predominant negative symptoms, concomitant psychiatric disorders or medical illnesses, and those done in first episode or stable patients. Published and unpublished studies were sought through database searches, trial registries and websites. Study selection and data extraction were conducted by at least two independent reviewers. Our primary outcome is the change in positive symptoms on a validated rating scale. Secondary outcomes include number of dropouts, overall and negative symptoms of schizophrenia, response, relapse, adherence, depression, quality of life, functioning and adverse events. Analyses were conducted in $\mathrm{R}$ within a frequentist framework. The risk of bias in studies has been evaluated using the Cochrane Risk of Bias tool and the credibility of the evidence will be evaluated using an adaptation of the GRADE framework to NMA, recommended by the Cochrane guidance. Subgroup and sensitivity analyses will be conducted to assess the robustness of the findings. The protocol of this review has been registered in Prospero (registration number: CRD42017067795).

Results: After screening 20196 references for title and abstract and 2555 full text articles, we identified 58 suitable trials, for a total of 3956 participants. Regarding primary outcome positive symptoms, only Cognitive Behavioural Therapy was significantly more effective than treatment as usual, with a standardised mean difference of -0.59 [95\% credible interval $-1.03 ;-0.16]$. The standardised mean differences with $95 \%$ credible intervals for other interventions were: Acceptance and Commitment Therapy -0.07 [-2.12; 1.98], Cognitive Therapy -0.18 [-1.92; 1.55], Hallucination Treatment -0.69 [-2.40; 1.01], Metacognitive Therapy -0.26 [-1.16; 0.64], Mindfulness -0.26 $[-2.14 ; 1.62]$, with heterogeneity $\operatorname{tau}^{2}=0.6942$.

Data analyses on other outcomes are ongoing.

Discussion: We are going to investigate the possible sources of heterogeneity with the pre-planned subgroup analyses: number of sessions, study duration, individual versus group setting, expertise of the therapist and baseline severity.

A network meta-analysis is the only methodology that allows the production of hierarchies of interventions for treatment of schizophrenia. Such hierarchies, saying which treatment is likely to be the best, the second best and so on, are essential for guideline development. The results of this study are therefore likely to provide knowledge of great impact for treatment decisions.

This project has received funding from the European Union's Horizon 2020 research and innovation programme under the Marie Skłodowska-Curie grant agreement No 701717.

\section{F215. EARLY SIGNS ACTION PLAN: EXPERIENCES OF RELATIVES}

Nils Sjöström*,, David Berg², Sofia Johansson ${ }^{1}$, Eva Andreasson ${ }^{1}$, Marda Waern ${ }^{2}$

\author{
${ }^{1}$ Sahlgrenska University Hospital; ${ }^{2}$ Sahlgrenska University \\ Hospital, University of Gothenburg
}

Background: The shift towards person-centered care is ongoing within healthcare today. The Early Signs Action Plan was developed to facilitate participation of patients and their next-of-kin in outpatient psychiatric services specialized in the treatment of persons with schizophrenia and similar disorders. The aim was to investigate relatives' experiences regarding the activation of the action plan for their next-of-kin.

Methods: The study is a qualitative interview study using a semi-structured interview guide. The interviews are conducted with relatives (anticipated $\mathrm{N}=10$ ) to outpatients whose Early Signs Action Plan has been activated. Interviews are digitally recorded and transcribed verbatim. The material is analyzed with qualitative content analysis

Results: Preliminary analysis based on the first six interviews suggests that relatives experience increased involvement in services as well as improved relations with care staff. Relatives felt a greater sense of security as they were more knowledgeable, and activation of the plan resulted in a more immediate response from service providers. However, some respondents expressed communication problems with staff, pointing to a need for improved flow of information and increased understanding of the situation. Some expressed a feeling of uncertainty related to lack of feedback from staff, as well as lack of continuity and limited inclusion in the care process. Results from the entire study will be presented

Discussion: Early Signs Action Plan may constitute a useful tool for the involvement of relatives in psychiatric services. However, relatives pointed out several areas for improvement

\section{F216. SLEEP QUALITY AND CLINICAL IMPROVEMENT IN FIRST EPISODE PSYCHOSIS}

\author{
L. Prayosha Villa ${ }^{1}$, Lyndsay Schmidt ${ }^{1}$, Tyler M. Moore ${ }^{1}$, \\ Monica Calkins, $^{*}$, Christian G. Kohler ${ }^{1}$ \\ ${ }^{1}$ University of Pennsylvania; ${ }^{2}$ University of Pennsylvania School of \\ Medicine
}

Background: Sleep disturbance is a common feature in early psychosis. Sleep quality has shown to be associated with both symptom severity and clinical improvement in persons with chronic illness.

Understanding the influence of sleep quality in early psychosis can be beneficial in determining interventions for coordinated specialty care (CSC). Using patients from a CSC intervention program for first episode psychosis, we investigated the association between subjective sleep quality with clinical response and clinical symptom correlates.

Methods: Participants were consecutive patients admitted between March 2015 to June 2017 who underwent coordinated specialty care at Penn PERC (Psychosis Evaluation and Recovery Center). Eligible participants were young persons ages 16-35 years who had experienced onset of psychosis within 3 years prior to intake and who underwent 2-years of CSC for early psychosis, including cognitive therapy for psychosis recovery (CT$\mathrm{R})$, medication management, family education and occupational support. Standardized self and observer based rating scales evaluating sleep quality (PSQI) and other clinical symptoms, e.g., anxiety (BAI), depression (BDI) and affective states (PANAS), and clinical improvement (CGI-I) were administered at intake, after 3 months, 6 months and subsequently every 6 months of CSC. Participants provided informed consent. 48 participants completed assessment at 2-time points between intake and 2-4 months later and 38 underwent assessment at 3 time points, including 6-7 months following intake. Correlational analyses were performed on PSQI change (slope) over 3 assessments and change in BAI, BDI, PANAS-negative, PANASpositive. Analysis were further stratified by improvement - CGI-I $<2$ (much improvement) $(\mathrm{n}=17)$ and CGI $>3$ (little/no improvement) $(\mathrm{n}=21)$.

Results: Of 48 patients, average age at intake was 22 years (Male:Female=40:8; Caucasian:African-American/Other=28:20). Primary 
analyses of sleep quality and clinical improvement included participants with three PSQI rating timepoints over 6-7 months of CSC $(n=38)$. Overall PSQI ratings did not change significantly over time. BAI and BDI-II scores significantly decreased over time, indicating subjective clinical improvement with treatment. There was a trend for positive correlations among PSQI, and BAI and BDI-II scores. When stratified by improvement, those rated 'much improved' group greater reduction of PSQI scores.

Discussion: We found that improved sleep quality was present in participants who experienced much global clinical improvement over 6 months of CSC. In addition, better sleep quality correlated with reduced depression and anxiety symptoms. Though these findings do not address direction of causality, our findings indicate that improving sleep quality should be a specific focus in treatment of early psychosis. Further analysis will be conducted to investigate the relationship between sleep and clinical improvement using other clinical measures, such as symptom severity, and the dataset will be expanded to include data through the end of 2017.

\section{F217. BASIC SELF-DISTURBANCE IN ADOLESCENCE AND SCHIZOPHRENIA- SPECTRUM DISORDERS IN YOUNG ADULTHOOD: A 7-YEAR FOLLOW-UP STUDY AMONG TREATMENT-SEEKING ADOLESCENTS}

\author{
Dan Koren*,1, Yair Tzivoni ${ }^{1}$, Liat Schalit ${ }^{1}$, Noa Reznik ${ }^{1}$, \\ Merav Adres ${ }^{1}$, Josef Parnas ${ }^{2}$ \\ ${ }^{1}$ University of Haifa; ${ }^{2}$ University of Copenhagen
}

Background: Phenomenological research indicates that disturbance of the basic sense of self may be a core phenotypic marker of schizophrenia spectrum disorders. Basic self-disturbance refers to a disruption of the sense of first-person perspective and self-presence that is associated with a variety of anomalous subjective experiences. Recent cross-sectional and prospective pilot studies provided preliminary support for the notion that SD may provide a means of further "closing in" on individuals truly at high-risk for psychosis, particularly of schizophrenia spectrum disorders (SSD). The goal of this study was to replicate and extend these pilot findings by examining the long-term persistence of SD and the degree to which their level in adolescence predicts SSD seven years later in young adulthood.

Methods: The 7-year stability of SD and their association with later in life SSD were explored in a sample of 40 young adults. SD was assessed with the Examination of Anomalous Self-Experience (EASE), prodromal symptoms and syndromes were assessed with the Structured Interview for Prodromal Syndromes (SIPS), present and lifetime diagnoses of schizophrenia-spectrum and other co-morbid disorders were assessed with the Kiddie Schedule for Affective Disorders and Schizophrenia (K-SADS) in adolescence and the Operational Criteria (OPCRIT) checklist for psychotic and affective illness in young adulthood, level of distress with the Mood and Anxiety States Questionnaire (MASQ), and psychosocial functioning with the Strength and Difficulties Questionnaire (SDQ).

Results: Forty young adults (Mean age $=23.7$, S.D. $=1.3$ ) out of the 82 who had participated seven years earlier in a study on the association between SD and attenuated psychosis symptoms (APS) were available and agreed to participate in the 1-year follow-up (Mean=1.4, S.D.=0.8). There were no significant differences between those who were available and those who lost for the follow-up assessment on any of the major socio-demographic or clinical variables at baseline. Eight $(20 \%)$ of the 40 participants in the present study met diagnostic criteria for an SSD (2 Schizophrenia, three nonorganic psychotic disorder, and three schizotypal personality disorder). The total EASE score was slightly higher in young adulthood compared to seven years earlier. However, this can reflect a difference in the administration method of the EASE between the two occasions. Consistent with our first hypothesis, the correlation between the total EASE score at baseline and 7 -year follow-up was moderate and significant $(\mathrm{r}=0.59, \mathrm{p}<.001)$. Similarly, consistent with our second hypothesis, SD at baseline was a significant predictor of an SSD diagnosis in young adulthood.
Discussion: These results provide further support for the temporal stability of SD over time. Also, they provide further support for the notion that SD is a phenotypic indicator of risk for SSD.

\section{F218. REAL-TIME ASSESSMENT OF AUDITORY HALLUCINATIONS USING A SMARTPHONE APPLICATION; RESULTS FROM A PILOT STUDY}

Josef Bless $^{*, 1}$, Runar Smelror ${ }^{2}$, Ingrid Agartz ${ }^{2}$, Kenneth Hugdahl ${ }^{3}$ ${ }^{1}$ University of Bergen; ${ }^{2}$ Norwegian Center of Excellence for Mental Disorders Research, University of Oslo, Diakonhjemmet Hospital;

${ }^{3}$ University of Bergen, Norwegian Center of Excellence for Mental Disorders Research, University of Oslo, Haukeland University Hospital

Background: A challenge in current research on auditory hallucinations (AHs) is that the assessment of symptom dimensions largely depends on structured interview scales, such as the PANSS, PSYRATS etc. In order to collect more ecologically valid data, we developed a smartphone app that can be used by patients to report on their experience in real-time, i.e. when the voices are actually present. The aim of this study was to investigate feasibility of the app and whether it can provide new phenomenological information on the temporal fluctuations of AHs in adolescent patients with early-onset schizophrenia (EOS).

Methods: Using the experience sampling method, one adolescent EOS patient used the app for a period of 16 days, during which the patient received random reminders five times per day, to answer questions on five dimensions relevant to AHs: Control (no - full), Content (negative - positive), Localization (outside head - inside head), Intensity (yelling - whispering), and Influence (not troublesome - very troublesome). The answers were registered on visual analog scales (VASs) implemented in the app.

Results: The patient responded to the notifications in $87 \%$ of the cases and in addition completed the questions 15 times on own initiative. In $73 \%$ of all responses, the patient indicated to experience AHs at the time of response. The results from the VASs showed that AH-dimensions are not stable but fluctuate over time. Several AVH-dimensions were significantly correlated $(\mathrm{p}<.01)$ with each other: Influence correlated with Content $(r=-.71)$, Intensity $(r=-.37)$, and Control $(r=-.76)$, whereas Content correlated with Intensity $(r=.39)$ and Control $(r=.57)$. showed several correlations a negative correlation with content of however, only localization (voices coming from outside - inside the head) correlated significantly with the number of days in use. In addition, the participant reported more internal voices over the course of 16 days $(p<.01$; $\mathrm{r}=.36)$ and later hours of the day $(\mathrm{p}<.05 ; \mathrm{r}=.22)$.

Discussion: The app captures the ebb-and-flow of AVHs and provides a unique profile of symptom severity and interrelationship between AVHdimensions. Such information has potential relevance for patient-tailored intervention.

\section{F219. NOVEL OBJECTIVE ASSESSMENT OF ACTIVITY ENGAGEMENT IN SCHIZOPHRENIA USING WIRELESS MOTION CAPTURE}

\author{
Ishraq Siddiqui*,1, Gary Remington ${ }^{1}$, Gagan Fervaha ${ }^{1}$, \\ Paul Fletcher ${ }^{1}$, Aristotle Voineskos ${ }^{1}$, Sarah Saperia ${ }^{1}$, \\ Konstantine Zakzanis ${ }^{2}$, George Foussias ${ }^{1}$ \\ ${ }^{1}$ Centre for Addiction and Mental Health; ${ }^{2}$ University of Toronto \\ Scarborough
}

Background: Amotivation and reduced engagement in goal-directed activities are prominent features of schizophrenia. Previous investigations of patients' engagement in activities have largely relied on accounts of 